\title{
Investigating human-robot cooperation in a hospital environment
}

\author{
Scrutinising visions and actual realisation of mobile robots in service work \\ Kristina, KT, Tornbjerg \\ Department of Planning, Aalborg University \\ kristinat@plan.aau.dk \\ Mikael B., MBS, Skov \\ Department of Computer Science, Aalborg University \\ dubois@cs.aau.dk \\ Anne Marie, AMK, Kanstrup \\ Department of Planning, Aalborg University \\ kanstrup@plan.aau.dk \\ Matthias, MR, Rehm \\ Department of Architecture, Design and Media \\ Technology, Aalborg University \\ matthias@create.aau.dk
}

\begin{abstract}
This study analysed work activity in a hospital basement where humans and robots interacted and cooperated on logistics tasks. The robots were deployed to automate parts of courier processes and improve the work environment for the hospital's kitchen staff. Human-robot cooperation was studied through ethnographic fieldwork relating to mobile service robots and hospital kitchen staff. The results highlighted problems arising through the assumption that the 'plug and play' service robots could effectively automate work tasks. The analysis revealed the complexity of human-robot interaction in dynamic work settings such as hospitals and identified contradictions between the envisioning and realisation of robots at work, as well as the visible and invisible procedures underpinning human-robot cooperation. Consequently, we emphasise the importance of considering robots as agents of change and draw attention to the new work practices that arise when robots assume the roles of workers in dynamic work settings.
\end{abstract}

\section{CCS CONCEPTS}

- Human-centred computing; • Human computer interaction (HCI); • Empirical studies in HCI;

\section{KEYWORDS}

Human-robot cooperation, hospital, service staff, invisible work

\section{ACM Reference Format:}

Kristina, KT, Tornbjerg, Anne Marie, AMK, Kanstrup, Mikael B., MBS, Skov, and Matthias, MR, Rehm. 2021. Investigating human-robot cooperation in a hospital environment: Scrutinising visions and actual realisation of mobile robots in service work. In Designing Interactive Systems Conference 2021 (DIS '21), fune 28-fuly 02, 2021, Virtual Event, USA. ACM, New York, NY, USA, 11 pages. https://doi.org/10.1145/3461778.3462101

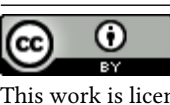

This work is licensed under a Creative Commons Attribution International 4.0 License.

DIS '21, fune 28-fuly 02, 2021, Virtual Event, USA

(C) 2021 Copyright held by the owner/author(s).

ACM ISBN 978-1-4503-8476-6/21/06.

https://doi.org/10.1145/3461778.3462101

\section{INTRODUCTION}

In many countries around the world, healthcare systems are changing and developing rapidly. Illnesses and diseases can be diagnosed and treated more effectively than ever before by, for example, using advanced technology and progressive medicine. Such developments are both necessary and appreciated because the ageing population in many countries places pressure on healthcare systems, and the outlook for pathological conditions involves an increasing number of patients with needs for hospital treatment, along with higher life expectancy and complex disease courses.[1] To meet the growing demand for superior healthcare services, hospitals must use their resources as effectively as possible by working smarter, optimizing their workflows, and minimizing non-productive activities. One promising way to achieve this is to assign repetitive, timeconsuming, and heavy tasks to robots, developed to perform such work quickly and effectively without the inconvenience of breaks, days off, or breakdowns. In general, the purpose of deploying robots in hospitals is to automate processes and procedures that were previously performed by humans, enabling humans to spend more time performing complex logistics tasks that robots are incapable of carrying out.

In hospitals, logistics processes involve many routine procedures that need to be maintained. These can be characterised as invisible work, since they are the underlying processes that generally go unnoticed. As pointed out by Star and Straus [2], the relationship between visible and invisible work is problematic. Lack of attention to tacit and contextual knowledge, acquired expertise, and teamwork can cause dramatic shifts in apparently stable work processes $[2,3]$; hence, research on human-robot collaboration in these types of invisible work settings is important for understanding and advancing work practices when robots take on the roles of workers in dynamic work settings.

There is a lack of work committed to understand the effects of deploying robots in work settings, but in recent years, human-centred perspectives on technology in the everyday life have received increasingly attention.

Researchers have argued for a new paradigm within HumanRobot Interaction (HRI), Critical Robotics (CR): a research field critically reflecting and researching how this technology is part of real-life settings. Researchers in CR [4-6] advocate for putting 
humans in the centre of HRI research and research robot technology holistically while focusing on values driven by technology $[7,8]$.

This paper contributes to the holistic focus in HRI research by investigating the use of mobile service robots in a real-life work setting and paying specific attention to understanding humanrobot cooperation on service tasks in a hospital basement. Our research aimed to explore implications of workers cooperating with mobile service robots, in order to gain understanding of robots in practice, in a rather under-investigated area, which can lead to shaping future cooperation between humans and technology in real-life settings.

\section{BACKGROUND}

\subsection{Logistic robots in hospitals}

Within healthcare settings, robots have been deployed to perform personal and professional tasks. Mettler and Raptis [10] distinguished between robots designed for use within clinical or care facilities and robots designed for nonclinical contexts, such as in patients' homes. The professional use of robots for healthcare further distinguishes between systems that are already deployed-such as robots for operations, surgical training, exoskeletons, prostheses and bionic limbs; therapy robots; assistive robots; telepresence robots, cleaning and disinfection robots; and logistic robots-and robots as manifestations of wishful thinking, such as robotic nurses and robots for keeping patients company. This study focused on logistic robots, which are often categorised as service robots since their purpose is primarily to assist humans with service tasks. [11] According to the ISO 8373 standard [12], a service robot is 'a robot that performs useful tasks for humans' and usually does so in cooperation with humans in dynamic environments (for example, hospital hallways) without surveillance or monitoring. Such robots have certain degrees of autonomy, which give them the ability to participate in daily routines and accomplish tasks (e.g. logistic robots handling transportation tasks) $[13,14]$. Logistic robots are often designed to attach to or carry carts, allowing them to transport food, laundry, and other items from one defined point to another. As presented in this paper, these robots can, for example, transport kitchen service items from a hospital kitchen to a ward and are intended to improve workflows and hospital effectiveness. Research on the potential of logistic robots in hospitals found that automation can improve logistics efficiency in hospitals, reduce costs, and facilitate organizational improvements. $[15,16]$

\subsection{Cooperating with logistic robots in hospitals}

Research advocating human-oriented approaches towards robots in real-life settings and aiming to understand humans and robots interacting and cooperating, has indicated that organizational factors impact the working relationship between the two parties. Wellbeing, including tolerance to interruptions and stress, has a great impact on how robots are experienced by the hospital staff who cooperate with such technology. Mutlu and Forlizzi's [17] ethnographic studies researched mobile robots in hospitals and found that different hospital wards perceived the same robot in different ways, due to varying levels of tolerance to interruptions: staff on a hospital's medical ward saw the robot as interrupting their work, while the staff on the post-partum ward had a less disruptive experience with the robot. Thus, the composition of a working environment influenced how hospital staff cooperated with robots, since the post-partum unit did not have as much traffic as the medical ward and did not deal with such urgent crises.

Human-centred studies of robots in hospitals have reported how human perceptions of robots in a workplace can change over time as human workers become more familiar with them. Ljungblad and colleagues [18] researched hospital staffs' perceptions of a robot transporting blood samples in an orthopaedic unit and found that perspectives on the robot adjusted over time as staff developed closer working relationships with the technology. These authors present a model on how the robot is often first perceived as something alien and machinelike and eventually as a coworker [18].

Mettler and colleagues [19] demonstrated that hospital staff cooperating with service robots had concerns about the complexity of the robots and the difficulties it might cause if this technology was integrated into hospital environments, claiming that some hospital staff were unable to think of robots as anything other than gadgets. Additionally, Cresswell and colleagues argued that humans' initially negative attitudes towards robots at the start of an exposure period are embedded in three major elements: lack of trust in the robots, fear of the robots threatening professional roles, and fear of the unknown [20].

Our research investigates how the mobile robots were respectively anticipated and used in practice in a hospital setting, aiming at understanding how robots and humans cooperate in dynamic work environments.

\subsection{Researching human-robot cooperation}

Although research on human-robot collaboration in dynamic shared work settings is scarce, research on collaboration on work mediated by technology is rich, especially regarding computersupported cooperative work (CSCW). As explained by Schmidt and Bannon [21], this research field is comprehensive, interdisciplinary, and heterogeneous, but characterised by a focus on problems relating to the design and use of technologies at work. Research attention has especially been paid to understanding coordinated practices at work $[22,23]$, drawing attention to assumed models of use [24] and a general need for 'systematic studies of actual cooperative work practices in real-world settings' [21] to unpack the practices that underpin the automation of work and highlight the situated character of work [25].

The phenomena and concepts of work have been widely debated in the CSCW research field, especially regarding whether 'work' only refers to paid work and highlighting that activities carried out in the private sphere are also work [26] (e.g. work relating to living with chronic illness) [27]. However, as emphasised by Schmith [28], the key focus of this line of research is on cooperative work, which is highly complex, often characterised by interdependencies, and 'requires and exhibits highly developed and often sophisticated coordinative practices of a nature that one rarely, if ever, finds outside of ordinary work settings' [28 p.392].

Our research was grounded in this perspective on cooperative work, paying specific attention to investigating not only formally defined work activities, but also conducting empirical research. 


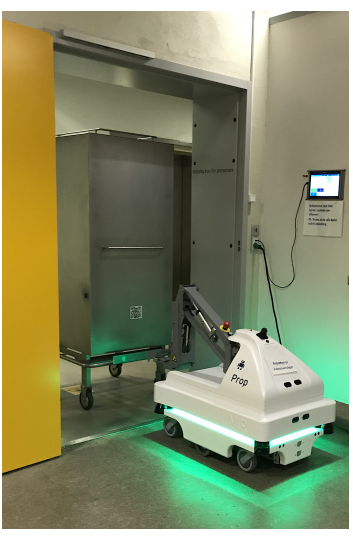

Figure 1: A MiR Hook 100 robot with a hospital kitchen service cart attached

The aim was to understand the cooperation between humans and robots on service tasks and discover the undefined, invisible work processes that often go unnoticed when technology is deployed to optimise workflows and facilitate shared tasks with humans.

The following Methods section presents the methods through which these phenomena were researched.

\section{METHODS}

This research investigated the complexity of human-robot work activity in a dynamic, shared environment in a hospital basement, where humans and robots interacted and cooperated on logistics tasks. The study was conducted in a medium-sized Danish hospital with approximately 3,000 employees across its operational region. The region covers a population of around 228,000 people, and at the unit level in this study, the hospital mainly carried out planned same-day surgeries, the treatment and monitoring of chronically ill citizens, and the treatment of injured locals and outpatients. The hospital deployed service robots to operate in the basement, where hallways are narrow and traffic (constituting a mix of patients, clinicians, laboratory technicians, kitchen staff, porters, technical staff, and workmen) is heavy.

\subsection{Robots}

In 2016, the hospital in this study deployed two mobile MiR Hook 100 TM service robots, which are autonomous, mobile service robots with an attached hook, developed to pick up and unload carts (see Figure 1).

This type of mobile service robot is widely used in industry, operating largely without human interaction, but these robots gradually gained acceptance in this hospital as a means of optimising logistics and were, consequently, interacting with humans in a dynamic environment in the hospital basement. These robots identified service carts that needed to be picked up from various hospital locations using QR codes and were equipped with sensor input, enabling the robots to evaluate the current setting and avoid driving into (human or inanimate) obstacles.
Furthermore, both robots had a large, red 'STOP button' that, when pushed, stopped the robots immediately in case of an emergency and a joystick for manual operation. The robots communicated with their surroundings using sound and light signals, which indicated the planned direction and the status of the current tasks they were performing.

\subsection{Informants}

Twenty-six people participated as informants in our study, all of whom were employed at the hospital, where most of them were kitchen staff $(n=16)$, two were kitchen service managers, one was a healthcare professional, and seven were porters, as shown in Table 1

The participants were recruited through snowball sampling [29]: The first author established contact with the hospital's kitchen manager, identified by members of the first author's professional network. The manager permitted access to the field, invited the hospital staff whose daily routines were based in the cellar to voluntarily participate in the research, and provided them with information about the study. The informants were then gradually recruited by the first author during the field study based on their interaction with the robots: the kitchen staff were included in the study because of their extensive cooperation with the robots, the healthcare professional was included because she impulsively interfered with one of the robots, and the porters were included because they had several interactions with the robots throughout the day. The informants also contributed to the observed traffic, along with the other humans in the basement, such as patients, clinicians, laboratory technicians, technical staff, and workmen. Throughout this paper, informants will be referred to by numbers such as Informant \#1 to ensure anonymity.

\subsection{Data collection}

We collected data through an ethnographic field study during May 2020. The methods used to gather empirical insights consisted of interviews based on an interview guide, observations of robots in action, and guided tours during which informants explained their actions when performing tasks and cooperating/not cooperating with robots [30, 31]. The aim of using a mixed-methods approach was to deepen understanding of robots in a hospital setting, observe the setting at first hand [32], investigate cooperation on tasks and procedures, explore the informants' visions of robot deployment, examine the actual everyday practices of robots at work, and uncover the hospital staffs' perceptions of working with robots $[33,34]$. Furthermore, the robots were shadowed by the first author during their performance of daily routine tasks [35]. The data was brought back from the field in the form of descriptive field notes, photographs and videos, and audio recordings of interviews.

\subsection{Data analysis}

The audio recordings of the interviews and guided tours (598 minutes in total $=9.97$ hours) were transcribed, the photographs and videos were examined, and the empirical data was analysed according to Braun and Clarke's techniques for thematic analysis [36]. 
Table 1: Overview of informants in the field study in the hospital

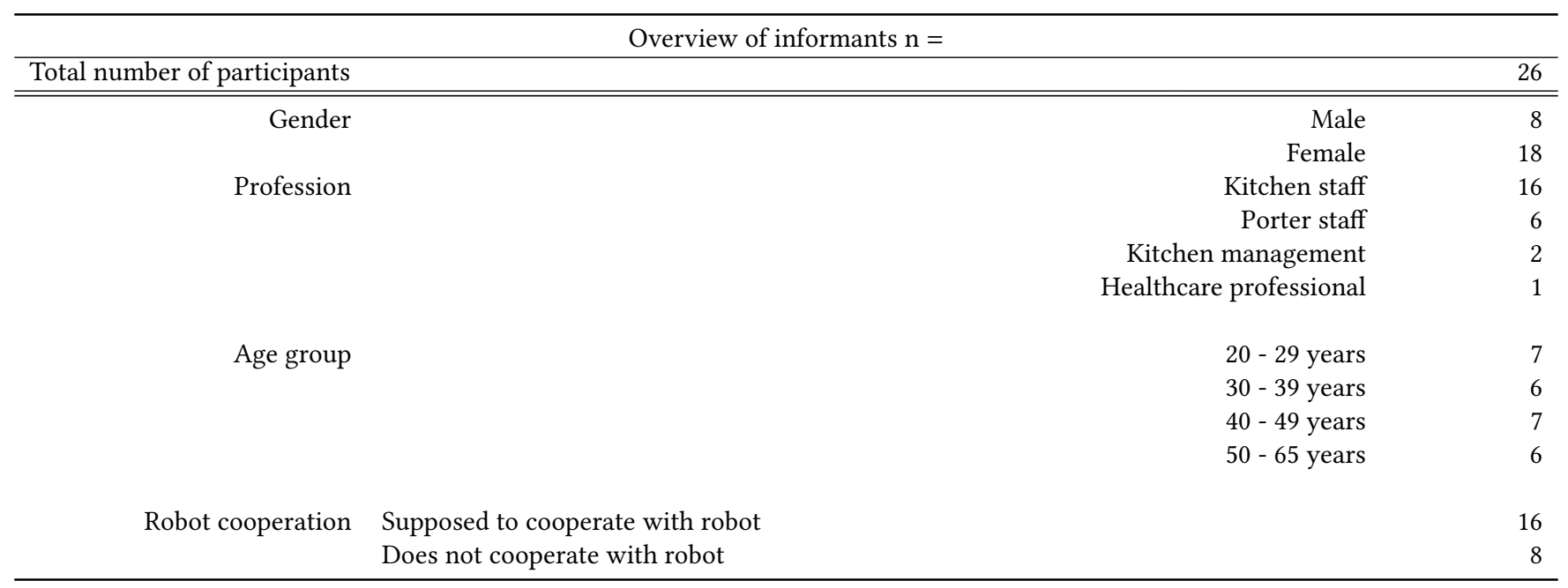

The transcripts and field notes were printed and colour-coded manually with markers. The coding resulted in 64 codes based on careful attention to identifying human-robot cooperation. The codes were organised into 12 themes: reliance, attitudes, errors, conflicts, pace of work, types of tasks, divisions of labour, perceptions, environment, irritation/frustration, 'war stories', workarounds, breakdowns, handovers, safety, and behaviour. The themes were reviewed to determine how the human-robot cooperation affected the work processes and procedures and how work was structured, planned, and carried out. In addition to the thematic analysis, Beyer and Holtzblatts' [37] work models were used to analyse the empirical data; identify the key aspects of work (such as strategies, roles, coordination, and structures); break the work processes down into steps to distinguish the robots' tasks from the humans' tasks; and identify the rich patterns of the agents' work. Flowcharts were designed, inspired by Jurgensen [38], to map and visualise how the work was divided, coordinated, and organised between humans and robots. The thematic analysis and flowcharts resulted in the identification of three key themes, which are presented in the following sections.

\section{FINDINGS}

\subsection{Robots in a hospital setting}

The key finding from the field study of robots in a hospital setting was that several obstacles presented challenges for integrating robots in a working environment. The hospital's kitchen manager explained that the aim of deploying robots was to ease the work of the kitchen staff following the implementation of a new food concept for patients. The hospital intended to provide patients with a higher quality food experience during their stays in the hospital and therefore deployed the robots to support staff in their daily work routines and relieve them of physical tasks, such as transporting carts with cutlery, glass, and dishes around the hospital. In an interview with the deputy kitchen manager, she explained this vision and shared how it was realised by the robots.

During the field study, the first author observed how the robot picked up a cart by using its hook, attaching itself to the cart, and pulled the cart around the basement to place it in another predefined spot, where the hospital kitchen staff could collect it, push it inside an elevator, and take it a hospital ward for use (see Figure 2). The robots' joysticks, located on their front casings, were a requirement of the hospital's management, enabling different hospital staff groups to manually drive the robots around in urgent cases, such as robots driving in front of staff handling emergencies. Additionally, it was possible to set the robots up in manual mode and control them through a specific tablet. When the robots were not running missions, they were parked for recharging in a room called the 'Robot Garage'.

The robots mainly performed tasks coded in a fleet management system (through which they functioned) and performed tasks/missions that the kitchen staff could define through a tablet located in the Robot Garage. Some kitchen staff informants $(\mathrm{n}=$ 8) showed how they gained an overview of the robots' locations through a map on the tablet, as long as the robots were operating within a certain distance from the tablet. If the robots went beyond this distance, the staff lost the connection and ability to track them, which necessitated them physically searching the hospital hallways for the robots. A kitchen staff informant reported that she and her colleagues were unable to locate the robots on the map if they drove out of range, if they had been turned off, or if they ran out of power (Informant \#7).

In the interview with the hospital's kitchen manager, she explained that she and the rest of the management team decided to give the robots names to ease the acceptance of the technology by different groups of hospital staff. They named the robots Prop and Berta, based on a popular Danish children's book and movie about a man (Prop) and his best friend (a cow called Berta). Prop and Berta only operated in the basement, because they were unable to fit into the hospital elevators when the carts were attached. Consequently, the robots only pulled service carts and did not transport supplies to hospital wards. The kitchen manager also explained in an interview that the decision to focus the robots' tasks on kitchen services was a result of the robots not being reliable enough for 

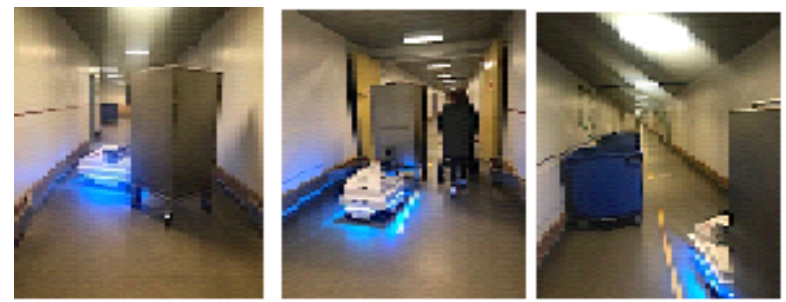

Figure 2: Mobile service robots driving in the narrow hallways of the hospital basement. The picture on the left illustrates how much space a robot with a cart attached took up in the hallway; the picture in the centre shows how a robot with a cart attached passed a patient in a wheelchair and her relative; and the picture on the right demonstrates how crowded the hallways in the basement could become, limiting available space.

the hospital management to charge them with critical tasks such as transporting blood.

In the hospital basement, the hallways were narrow and the robots were therefore programmed not to drive alongside each other. As shown in Figure 2, the hallways were not suitable for several types of traffic at the same time; hence, the robots could only navigate around humans with a certain amount of surrounding space available (for safety reasons), and they took wide turns for mechanical reasons. A consequence of the narrow hallways combined with the wide robot movements was that the robots took up a great deal of space in the hallways when working.

\subsection{Visions for and actual realisation of the robots' work}

Through the study, it became clear that several issues affected the cooperation between hospital staff and robots, which staff members had to deal with daily. In the interviews and observations, it was evident that the vision of simple automation being able to handle work tasks and procedures was unrealistic in practice. During an interview with the kitchen leader, she outlined that the vision of deploying mobile service robots in the hospital was to save time and optimise the work processes of the hospital kitchen staff: 'In my opinion, the robots are definitely giving great aid to the kitchen staff, who would have been unable to accomplish the considerable number of tasks they have without the robots' automated performance' (Informant \#18).

According to the kitchen leader, the robots were deployed following the new meal concept in the hospital, which had to be implemented without additional resources, except the robots, being given to the kitchen. She explained that, consequently, tasks that could not be completed by the current staff had to be automated and performed by the robots, constituting robot labour as an inevitable factor in the daily work processes. The deputy kitchen manager elaborated on how the robots were supposed to relieve the kitchen staff of routine work and improve their work environment, saying: 'Our kitchen staff are no longer supposed to push and pull the heavy service carts across long distances in the hallways of the hospital. Now they only have to make minimal physical effort in the task of transporting stuff' (Informant \#17). However, only a single courier task was performed by robots: transporting service carts from one identified spot to another. This observation is illustrated in Figure 3. To enable a robot to pick up a cart and transport it, the kitchen staff had to collect a cart from the kitchen (located on the ground floor), take it down to the basement in an elevator, and place it in a certain spot, such as the Robot Garage or the hallway. This procedure was necessary because the robots' size made them incapable of fitting into the elevators when carts were attached. From that spot, the robot could collect a cart and transport it to another destination (e.g. Temporary Destination A), where a member of the kitchen staff would take the cart and transport it to Final Destination B, which was usually located on the higher floors of the hospital (Figure 3).

This finding highlighted that the hospital's kitchen staff actually acquired a new type of task after the robots were deployed: placing the service carts in certain locations for collection by the robots.

Additionally, an unexpected finding was that 5 of the 16 kitchen staff members were following the robots around when they were transporting carts. These informants were spending time taking care of the robots and aiding them in fulfilling tasks. When interviewed, the staff members explained that they followed the robots in case something went wrong during the robot's mission, and all five informants pointed out that they did so because they had previously noticed errors when the robots were performing tasks. Hence, the study found that 'minding' the robots was also a new type of task for the kitchen staff following the deployment of the robots. These findings raised important questions about the implications of deploying robots to optimise work processes, since the empirical data showed that, although the hospital's kitchen staff were relieved of heavy work tasks, they spent considerable amounts of time assisting the robots.

Another important finding that unfolded during the interviews was that $50 \%$ of the kitchen staff informants preferred to work without interacting with the robots. Eight of the sixteen informants who were supposed to share the daily courier tasks with the robots explained that they preferred to push/pull the service carts around the hallways themselves because they could not rely on the robots to fulfil these tasks. Consequently, they felt it was more effective to perform tasks themselves, and one of them said: 'Something always goes wrong. Errors occur and the robots do not do as they are told, so we can't rely on them to accomplish their missions. If I want something done, I will do it myself' (Informant \#5). For this reason, we referred to these informants as non-users. Six of the eight non-users explained that, apart from their unreliability, they did not want to use the robots during their daily work routines because the robots slowed down their work. These six informants stressed that this happened because the robots ran slowly, not least when they took time to assess their surroundings for (as the informants said) uncertain reasons. This observation was important since it highlighted conflicts in balancing the work tempo in the cooperation between humans and robots. Furthermore, the nonusers stated that they could work faster themselves and did not consider the heavy pulling/pushing to be a problem; rather, the robots could be a source of frustration due to errors that influenced the work hours. One of them said:

Honestly, the robots are more a source of irritation than a helping hand! Don't get me wrong; it is fine when they are working without 


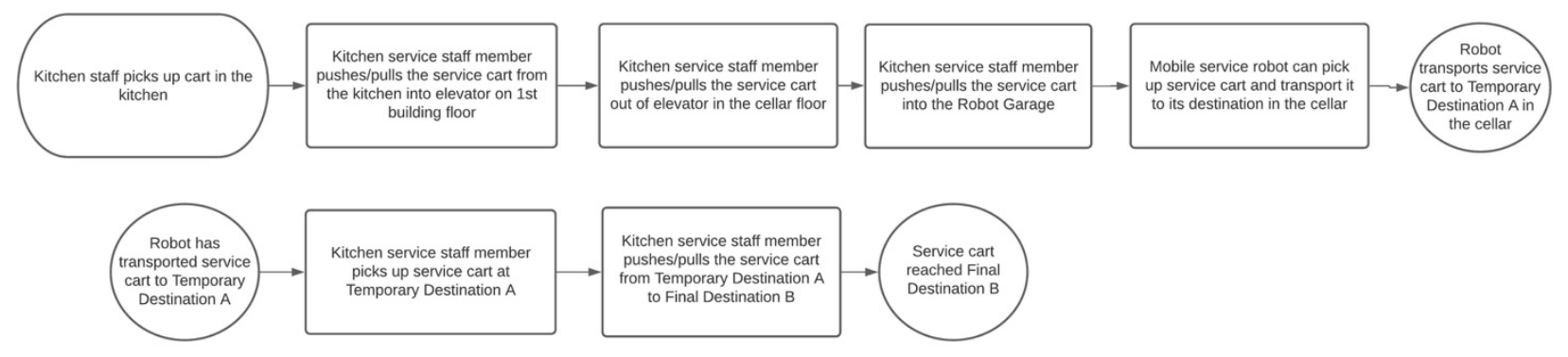

Figure 3: A visualization of the observed steps for transporting a service cart from one spot to another

problems, but errors occur so often ... It really can be a waste of time to use Prop and Berta, you know? (Informant \#2).

This was a key insight into how the kitchen management's visions for the robots as a mean to optimize the work procedures were not shared by the kitchen staff, who perceived the robots as a complicated and time-consuming source of extra work.

The study also found that the robots only helped kitchen staff to transport service carts carrying glass, cutlery, and dishes, since the staff still pushed/pulled carts containing food around the hospital. In an interview with the hospital's kitchen leader, she said that transporting food was not a task the robots could carry out, since the hospital had found robotic operations to be unstable. She elaborated that she and her colleagues found the robots unreliable for completing critical tasks.

Taken together, these findings supported our developing understanding of how humans and robots work together by illustrating why the visions of robots working in the hospital were difficult to achieve and why the realisation of cooperation between the kitchen staff and the robots was complex, leading to changes in the work rather than minimising work: the hospital kitchen staff acted as caretakers for the robots, despite the robots being deployed to carry out tasks and cooperate with the staff.

\subsection{Visible and invisible procedures in human-robot cooperation}

Through interviews and observations, it became clear that the procedures aiming to facilitate cooperation between kitchen staff and robots were complicated because the accomplishment of tasks comprised a series of invisible process $s$ and interactions between the robots and the dynamic work environment. To carry out the daily work routines of the hospital kitchen, tasks were divided, coordinated, and distributed between the kitchen staff and the mobile service robots by the kitchen management. In an interview with the kitchen's deputy manager, she explained how the formal division of labour was assigned and clarified why it was a simple task for a robot to move a service cart from point A to point B: the robot would simply drive up to the cart, attach itself to it, and move it from point $A$ to point $B$, as shown in Figure 4

In this workflow, the robot was sent on mission and drove off to accomplish it by moving down the hallway, approaching and attaching a cart, and then simply bringing the cart back to the Robot Garage. These processes were apparently simple, but guided tours with kitchen staff and robots showed that several steps and procedures for performing these tasks were unspecified, invisible, and informal. It became clear that the robotic workflows were far more complicated than envisaged by the kitchen's management and kitchen staff informants. Figure 5 visualises the steps observed during a single tour following a robot, which was performing the task of transporting a service cart from one point to another in the basement. Including a total of 28 different steps, in sharp contrast to the verbally reported 7-step workflows, this visualization presents an overview of several procedures and interactions that were invisible in the formal workflow shown in Figure 4, drawing attention to why robotic task performance in a dynamic environment must be considered as complex, comprehensive, and context dependent, rather than a simple, isolated automation of tasks.

As visualised in Figure 5, when a robot was sent on a mission by a member of the kitchen staff, the robot drove away from the Robot Garage and into the hallway extremely slowly to avoid crashing into the narrow door frame. The robot would then drive into the hallway and enter an environment that held mixed traffic; therefore, the robot would loudly announce its presence using alerting sounds and flashing lights (steps 1-5). Steps 6-11 visualise how a porter approached a robot too closely, which made the robot stop and the porter furious, since the robot was slowing down his work. In step 13, a healthcare professional pulled out in front of the robot with the consequences shown in steps 14-19. In step 14, the robot stopped as soon as its sensors registered movement and then started to play loud, alerting sounds ('Please step back! Please step back! Please step back!'), with lights flashing, to warn people in the surrounding area. The healthcare professional started to express her frustration by swearing at the robot, and because the robot was immobile, still warning and recalibrating, a member of the kitchen staff approached the robot with a cart full of warm meals for patients that she was on her way to deliver (step 17). Unfortunately, she was unable to pass the robot, which had stopped in the middle of the narrow hallway, blocking traffic. Consequently, the kitchen staff member and the healthcare professional had to wait for the robot to recalibrate and drive on before they could continue with their tasks. However, they failed to recognise that the robot would not have stopped if the healthcare professional had not moved in front of it. After five minutes, the robot was ready to proceed (step 20), drove on, and then attached the service cart to its hook, making a wide turn that again blocked the narrow hallway, before driving on towards the 


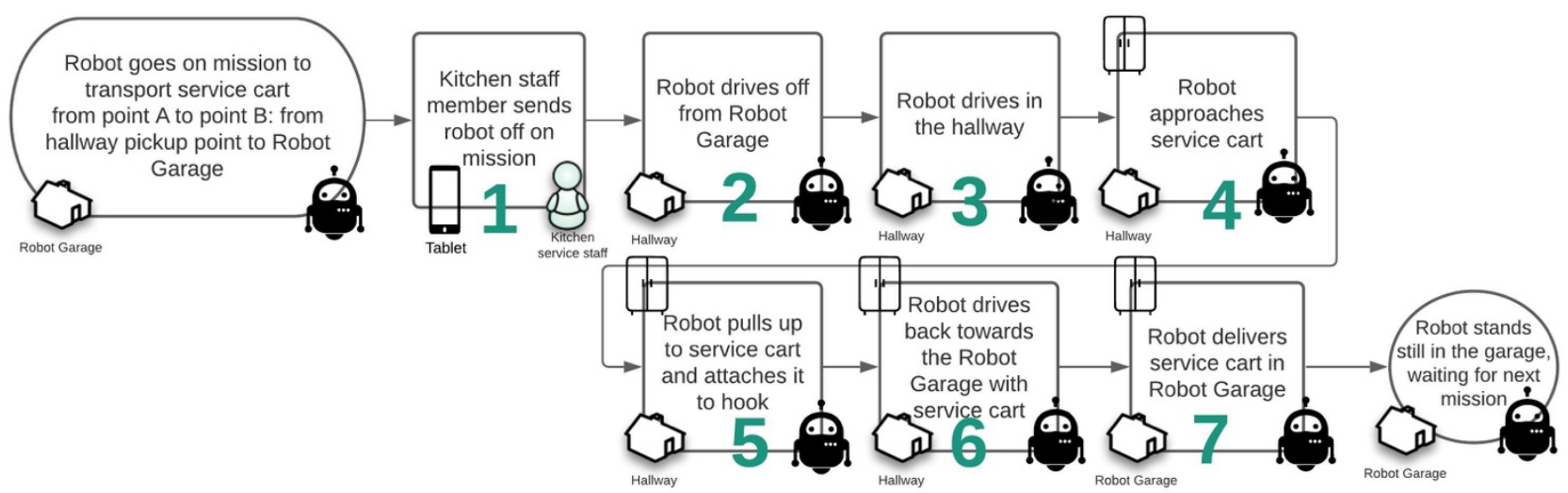

Figure 4: A visualization of the steps for a mobile service robot to perform a task, as expected and described by the kitchen's deputy manager

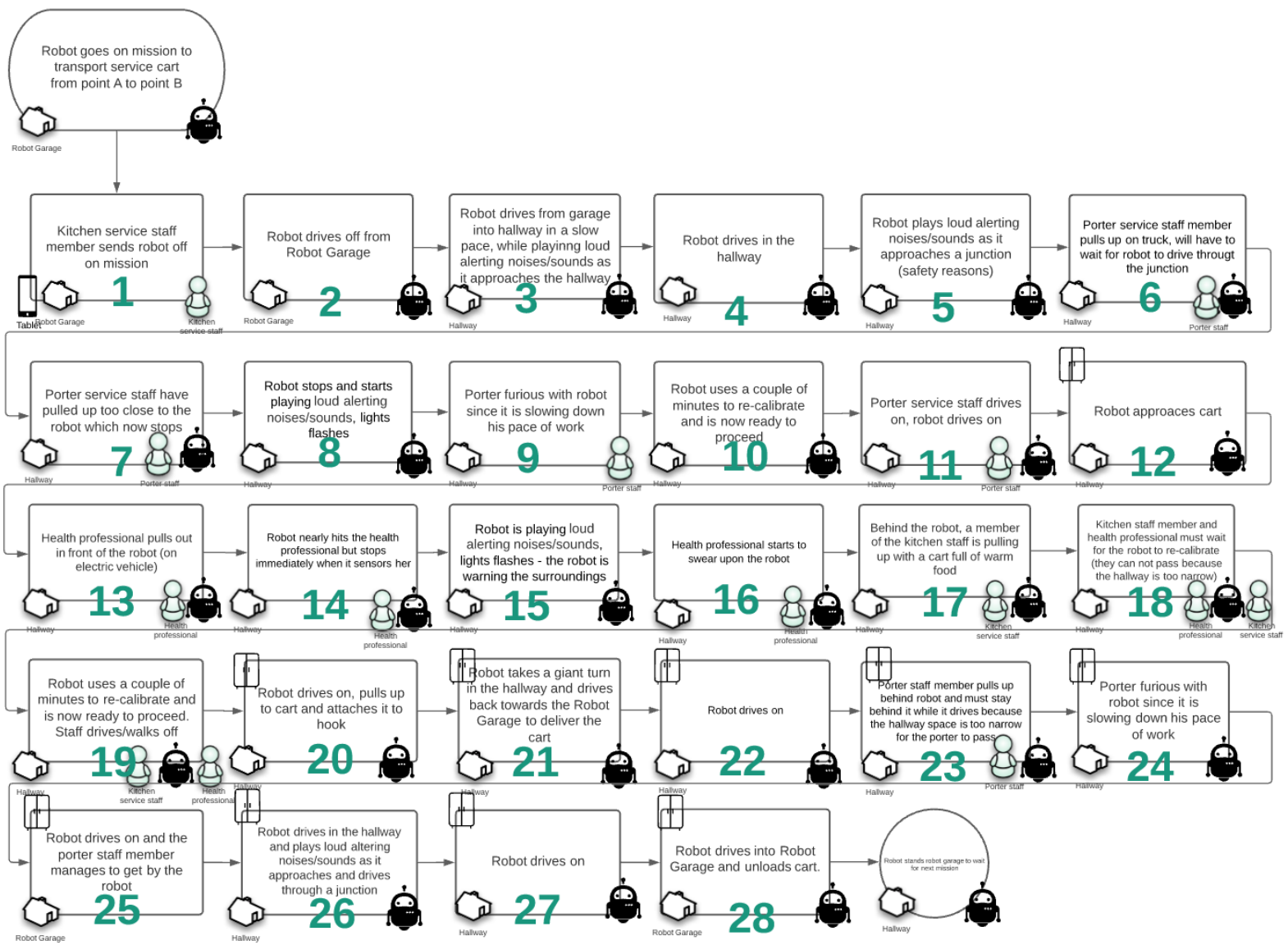

Figure 5: A visualization of the steps observed during a tour with one of the mobile service robots performing a task 
Robot Garage to unload the service cart. In steps 23-25, another porter pulled up, in a truck, behind the robot and had to drive behind it, which made him angry because the robot was forcing him to slow down. In steps 26-28, the robot drove on and arrived at the Robot Garage, where it unloaded the service cart. When it had done that, the kitchen staff members were able to take the service cart to its final destination.

These invisible procedures developed because unexpected interactions between humans and robots turned out to greatly affect the everyday cooperation between kitchen staff and robots, but the staff were not aware of the complexity of the processes. Through interviews, the kitchen staff informants were asked to elaborate on how the robots completed tasks, and only 2 of the 16 members of the kitchen staff were aware of how many processes the robots had to execute during their missions. This finding implies that the cooperation between humans and robots is complex, since robots are deployed, and expected, to perform seemingly simple tasks, but the cooperation between staff and robots depends on a comprehensive and invisible set of interactions, adding to the complexity of deploying robots for new applications, such as in hospitals.

\section{DISCUSSION}

This field study revealed a series of empirical situations that highlighted the complexity of integrating robots into dynamic work environments and the problems that arise when robots' deployment leads to less-than-seamless automation of tasks and processes. Moreover, this research showed that simple automation's idealised ability to handle work tasks and procedures is unrealistic in practice because of three major elements. First, the studied robots changed the staff's work, rather than minimising their work. Second, the procedures which aimed to facilitate cooperation between kitchen staff and robots were invisible. Third, humans' cooperation with robots in the studied context was complex.

In the following section, we discuss these findings and recommend a holistic, ecologic view when introducing robots to dynamic work settings. This view must consider not only the staff who are expected to cooperate with robots but also other stakeholders, the environment, and similar factors.

\subsection{Robots changing rather than minimizing work}

Technological deployments-in this research case, robots-in work settings can change the nature of work, tasks, and effort. The robots we researched had been installed at a hospital to optimise work processes and relieve kitchen staff of heavy daily tasks. Our empirical study highlighted how these hospital kitchen staff members acted as caretakers for the robots and how these robots imposed additional tasks on the staff, rather than taking over tasks as intended to optimise work processes and save time. For example, the hospital kitchen staff took on new types of tasks after the robots' deployment, such as placing service carts in certain spots around the hospital for the robots to pick up. Generally, the kitchen staff spent a great amount of time helping the robots fulfil tasks. Consequently, the kitchen staff did not share the kitchen managers' vision of the robots optimising work procedures; rather, the staff perceived the robots as a complicated, time-consuming source of extra work.

Altogether, our findings showed how robots necessitate changes to everyday work routines, rather than minimising human workloads or replacing the human workforce.

\subsection{Complicated cooperation due to invisible processes}

Our research showed that cooperation between humans and robots is complicated because tasks required a series of invisible processes and interactions between the robots and elements of the dynamic work environment. The everyday challenges between kitchen staff and the mobile robots contrasted with managers' perspectives on these robots. Neither hospital managers nor kitchen managers seemed to completely understand the complexity of kitchen staff and the robots' tasks because many steps were invisible or informal. Managers may have expected the robots to be able to perform tasks automatically, but when this study identified tasks' steps and procedures, the actual work practices were exposed, and the informal practices that supported the work of both kitchen staff and robots became clear.

As our findings revealed, such invisible procedures result in unforeseen interactions between humans and robots. In our studied case, such unanticipated interactions greatly affected everyday cooperation between the kitchen staff and the robots as part of the hospital basement's dynamic work setting.

\subsection{The complexity of deploying robots in a dynamic work setting}

The cooperation between staff and service robots is complex. In this study's case, this cooperation was complex because the robots are expected, to some extent, to autonomously perform simple tasks, but their cooperation with staff depended on a comprehensive set of interactions of which managers were unaware. These interactions made the robots and staff interdependent, adding to the complexity of deploying robots to new applications, such as hospital work. Technological deployments in work settings affect the nature of work, as Mutlu and Forlizzi have shown [17]. They found that a work environment's composition influences cooperation between hospital staff and robots. In the same vein, our research revealed complications in robots' daily work tasks because of environmental factors, such as the presence and actions of other basement stakeholders (e.g. porters and kitchen staff). These complications led to a lack of trust in the robots among the kitchen staff, and some staff members consequently avoided working with the robots despite managers' expectations that staff would cooperate with the robots. This finding supported the work of Cresswell and colleagues, who asserted that negative human attitudes towards robots are embedded in a lack of trust in robots and a fear that robots will threaten jobs [20].

Our research contributes to the body of knowledge regarding coordinated work practices by elucidating automation-related practices and explaining the potential complexity of robots' deployment to shared dynamic work settings. Some of the challenges that occurred while the robots performed tasks were inherently technological (e.g. the robots' slowness and safety measures that 
made the robots frustrating for porters and other humans working in the hospital basement), but the core issue with the robots was their protracted process of integration into workflows in order to seamlessly cooperate with kitchen staff. This process remained ongoing years after the robots had been deployed to the hospital.

The kitchen staff did not seem to have adjusted positively to the robots' presence, unlike the hospital staff in Ljungblad and colleagues' research [18], despite the robots' having been installed at the hospital in 2016. In our research, the staff did not seek closer involvement with the robots because they doubted the robots' reliability in performing tasks. According to the hospital's kitchen manager, no guidelines established how cooperation between kitchen staff and robots could be achieved, nor were any descriptions available of appropriate workflows or cooperation processes. Therefore, complementary work tasks between staff and robots were difficult to delegate or coordinate. When work was distributed between humans and robots, tasks' complexity was not taken into account because a significant number of steps in human-robot cooperation were unknown to the kitchen manager who was delegating these tasks. This manager was unaware of the processes and steps required to perform tasks in the basement work environment.

\subsection{Implications}

Since this study exposed disparate beliefs about human-robot interaction and cooperation in a dynamic hospital work setting, we emphasise the need to think of robots as a new technology, rather than a familiar and tested technology which can be simply adjusted and applied. Implementing robots in real-world work environments requires more nuance than plugging in the technology and pressing a 'power' button. In the same vein, technology must be developed beyond 'one size fits all' solutions because work environments vary. The mobile robots deployed to the hospital in this study were of a type that had been widely fostered and used in industry, moving items from Destination A to Destination B in highly structured environments, where humans working within a certain range of the robots are instructed and educated in sharing workspace with such. Since this kind of use and training are not matters of course when robots are implemented into hospitals, the humans who share their much more complex, unstructured work environment with mobile robots experience several problems in cooperating with this technology, as in industry cases. If robots are to participate in real-world work environments and cooperate with humans, sociotechnical factors must be focal - not least because humans affect the environments to which robots must adapt. This focus is complex, though, since robots do not hold any degree of social (or artificial) intelligence and are, therefore, unable to adjust their behaviours, work, and routines to the humans around them.

Important prerequisites for the successful deployment of robots in work settings are broader perspectives on which work elements this new technology affects and increased awareness of robots' requirements to function effectively in dynamic environments while closely cooperating with humans. Based on this field study's findings, we suggest a holistic perspective on robots in the same vein as 'the ecological viewpoint' defined by Nardi and O'Day [39] as 'a system of people, practices, values, and technologies in a particular local environment' [39, p. 49]. We, therefore, propose a consideration of robots in relation to 'a dense network of relationships in local environments' (i.e. information ecologies) [33, p. 27], which implies including robots as part of an ecosystem and preventing their exclusion from their surroundings. This ecological viewpoint would promote an understanding of optimal cooperation and interactions between different groups of hospital service staff and robots. For example, the routines and workflows of the porters in this study did not seem to have been integrated into the robots' workflows and organisation after their deployment in the hospital; nor did managers consider or act upon the porters' attitudes towards the robots - despite the robots having significantly affected the porters' performance of their tasks. If the porters had been involved in the deployment processes and given an opportunity to influence the robots' inclusion in their shared work environment, we presume the porters would have experienced less frustration with the robots' presence in the basement.

In order to introduce an ecological, holistic viewpoint at the hospital we studied, discerning the invisible aspects of work would be beneficial. If even some of these steps in the robots' task performance had been clear, managers could have evaluated them, preferably in close collaboration with the stakeholders and professionals whose daily work took place in the basement. Managers could, thus, have understood what the robots could contribute to the context - the ecology - in which they were deployed. This involvement would have allowed stakeholders and professionals to discuss their routines, values, work performance, needs, and desires vis-à-vis the robots, enabling the robots' deployment to be closely tailored to their expected work context work.

\subsection{Limitations and future work}

In this study, we chose to limit our research to one single robot type, mobile MiR100 robots, in a single and specific work environment. We chose this focus and limitation because this type of robot had been deployed for the longest period at the hospital where we collected our study's data. Therefore, the findings we have presented might not apply to cooperation between other types of robots and humans in hospitals. However, our findings must be considered empirical material which provides insights into human cooperating with MiR100 mobile robots within a service domain.

This ethnographic field study of mobile service robots at work has improved the broader understanding of the complexity in considering human-robot cooperation within a dynamic work setting. Our findings highlight the importance of understanding broader work settings and human-robot interactions when implementing robots into work, calling for further investigation of the dynamics between humans and robots in complex workplace settings which are not designed for robots. This further research should include sociotechnical research into robots' impact in a context that holds several interdependencies, tasks, procedures, interests, and stakeholders. The acknowledged principles for stakeholder involvement are greatly important to human-robot interactions, and future research is needed on understanding and designing optimal types of human-robot interactions for multiple stakeholders in hospital service work. Our research has illustrated why managers' visions of deploying robots in a work setting were not fulfilled in reality. 
When robots share work environments with humans, such visions seem to reflect high hopes among techno-enthusiasts and managers, but our research has shown a need to investigate the humans who actually interact and cooperate with robots in dynamic work environments in order to responsibly implement robots at work.

\section{CONCLUSION}

We investigated actual human-robot work activity in a hospital basement and identified how robots led to work changes, how invisible procedures caused complications, and why human-robot interaction and cooperation were complex. Our research has shown why the process of deploying robots to cooperate with humans must be acknowledged beyond the simple automation of work tasks. We have demonstrated that robots are agents of complex work changes and must be considered as such for any meaningful cooperation between robots and staff.

This research found that mobile service robots at the studied hospital were not considered reliable enough for critical tasks. Also, because they drove around the hospital basement with service carts, they took up a great deal of space, which especially frustrated porters and other staff members whose daily work routines had been disrupted.

This study has also identified complex cooperation between kitchen staff and robots. Our observations showed that everyday cooperation between kitchen staff and robots involved several problems - not least because a considerable amount of procedures involved invisible steps of work and different interactions between robots and the dynamic work environment. Our research gradually clarified that managers' expectations in deploying these robots to achieve simple, technical automation of work tasks and procedures was unrealistic in practice. The robots actually increased the kitchen staff's tasks, rather than optimising their workflows - not least because the kitchen staff tended to follow the robots around to ensure that they performed their tasks correctly, or the staff simply carried out the robots' tasks themselves. The hospital kitchen staff tended to become caretakers for the robots despite the robots' purpose of accomplishing tasks and cooperating with staff. Altogether, these findings provide an understanding of the key factors which underpin the complexity of robotic aid and assistance, revealing how robots necessitate changes in work routines, rather than replacing the human workforce.

Our findings in this study have revealed an apparent need to consider robots from holistic and ecological perspectives when designing them for, and deploying them in, work settings. Based on our findings, we posit that scrutinising robots in relation to their work surroundings and contributions would be beneficial.

\section{ACKNOWLEDGMENTS}

We thank all of our participants at The Hospital of Southern Jutland, Southern Region, Denmark, for their engagement in this study.

\section{REFERENCES}

[1] Danske Regioner. 2015. Pres på sundhedsvæsenet: Derfor stiger sygehusudgifterne - sådan holder vi væksten nede. Retrieved February 10, 2021 from https://www.regioner.dk/media/2209/2015-pres-paa-sundhedsvaesenet.pdf

[2] Leigh Star, Strauss. 1999. Layers of Silence, Arenas of Voice: The Ecology of Visible and Invisible Work. In Comput. Support. Coop. Work (CSCW), Vol. 9, Kluwer Academic Publishers, Netherlands.
[3] Lucy A. Suchman. 1987. Plans and situated actions: the problem of humanmachine communication. Cambridge University Press, USA.

[4] Sara Ljungblad, Sofia Serholt, Wolmet Barendregt, Pamela Lindgren, and Mohammad Obaid. 2016. Are We Really Addressing the Human in Human-Robot

[5] Selma Šabanović. 2010. Robots in society, society in robots. Int J of Soc Robot 2, 4: 439-450.

[6] Ylva Fernaeus, Mattias Jacobsson, Sara Ljungblad, Lars Erik Holmqvist. 2009. Are we living in a robot cargo cult? In Proceedings of the ACM/IEEE International Conference on Human-Robot Interaction (HRI' 09), 279-280.

[7] Donald MacKenzie and Judy Wajcman. 1999. Introductory essay: the social shaping of technology. In The Social Shaping of Technology (2nd. ed.), Donald MacKenzie and Judy Wajcman (Eds.). Open University Press, Buckingham, UK, 3- 27

[8] Sara Ljungblad, Sofia Serholt, T. Milosevic, Rikke Toft Norgaard, N. Ni Bhroin., P. Lindgren, C. Ess,W Barendregt, \& M. Obaid .2018. Critical Robotics - Exploring a New Paradigm. In Proceedings of the 10th Nordic Conference on HumanComputer Interaction (NordiCHI'18), Oslo, Norway.

[9] Sara Ljungblad, Sofia Serholt, Wolmet Barendregt, Pamela Lindgren, and Mohammad Obaid. 2016. Are We Really Addressing the Human in Human-Robot Interaction? Adopting the Phenomenologically- Situated Paradigm. In J. Seibt, M. Nørskov \& S. Schack (Eds.) What Social Robots Can and Should Do: Proceedings of Robophilosophy 2016/TRANSOR 2016. IOS Press, Amsterdam, Netherlands, 99- 103.

[10] Tobias Mettler, Dimitri Raptis. 2011. What Constitutes the Field of Health Information Systems? Fostering a Systematic Framework and Research Agenda. Health informatics journal. 18. 147-56.

[11] Tamás Haidegger, Marcos Barreto, Paulo Gonçalves, Maki K. Habib, Sampath Kumar Veera Ragavan, Howard Li, Alberto Vaccarella, Robert Perronea, and Edson Prestes. 2013. Applied ontologies and standards for service robots. Robot. Auton. Syst. 61, 11, 1215-1223.

[12] International Organization for Standardization. 2012. Robots and robotic devices - Vocabulary (ISO Standard No. 8373:2012. https://www.iso.org/obp/ui/fr/\#iso: std:iso:8373:ed-2:v1:en

[13] Jake Deery. 1997. Courier robot keeps hospital staff'on the job. The Journal for Healthcare Quality (JHQ): January-February, Volume 19, Issue 1, 22-23.

[14] TE Kirschling, SS Rough, BC Ludwig. 2009. Determining the feasibility of robotic courier medication delivery in a hospital setting. Am 7 Health Syst Pharm. 66(19):1754-1762

[15] MD Rossetti, A Kumar, RA Felder. 1998. Mobile robot simulation of clinical laboratory deliveries. Winter Simul. Conf. Proc., vol. 2, no. 1987, 1415-1422.

[16] J Evans, B Krishnamurthy, B Barrows, T Skewis, V Lumelsky. 1992. Handling real-world motion planning: a hospital transport robot. IEEE Control Systems Magazine, vol. 12, no. 1, 15-19.

[17] Bilge Mutlu, Jodi Forlizzi. 2008. Robots in organizations: The role of workflow, social, and environmental factors in human-robot interaction. Proc. 3rd ACM/IEEE International Conference on Human-Robot Interaction. Living with Robot. 287294.

[18] S Ljungblad, J Kotrbova, M Jacobsson, H Crame, K Niechwiadowicz. 2012. Hospital robot at work: Something alien or an intelligent colleague? Proc. ACM Conf. Comput. Support. Coop. Work. CSCW, no. February, 177-186.

[19] Tobias Mettler, Michaela Sprenger, Robert Winter. 2017. Service robots in hospitals: new perspectives on niche evolution and technology affordances, European Journal of Information Systems, 26:5, 451-468.

[20] K Cresswell, S Cunningham-Burley, A Sheikh. 2018. Health Care Robotics: Qualitative Exploration of Key Challenges and Future Directions. Journal of medical Internet research, 20(7), e10410.

[21] Keld Schmidt \& Liam Bannon. 2013. Constructing CSCW: The First Quarter Century. In Comput. Support. Coop. Work (CSCW) 22:345-372.

[22] Paul Luff, Jon Hindmarsh, Christian Heath. 2000. Workplace Studies: Recovering Work Pratice and Informing System Design. Cambridge University Press.

[23] Christian Health \& Paul Luff. 2000. Technology in Action. Cambridge University Press, 2000.

[24] Tom A Rodden, John A. Mariani, Gordon Blair. 1992. Supporting cooperative applications. Computer Supported Cooperative Work (CSCW): An International Journal, vol. 1, no. 1-2, 1992, pp. 41-68.

[25] Lucy Suchman: "Plans and situated actions: The problem of human-machine communication". Cambridge University Press, 1987.

[26] A Crabtree, TA Rodden, SD Benford. 2005. Moving with the times: IT research and the boundaries of CSCW. Comput. Support. Coop. Work (CSCW): The Journal of Collaborative Computing, 14(3), 217-251.

[27] Juliet Corbin, Anselm Strauss. 1985. Managing chronic illness at home: Three lines of work. Qualitative Sociology 8: 224-247.

[28] Keld Schmith. 2011. The Concept of 'work' in CSCW. Comput. Support. Coop. Work (CSCW) 20:341-401.

[29] Patrick Biernacki, Dan Waldorf. 1981. Snowball Sampling: Problems and Techniques of Chain Referral Sampling. Sociological Methods and Research. 10:141163.

[30] James P Spradley. 1979. The ethnographic interview. Holt, Rinehart and Winston, New York, USA. 
[31] Michele Everett, Margaret Barrett. 2012. Guided tour: A method for deepening the relational quality in narrative research. Qualitative Research Journal. 12. 32-46.

[32] Jeanette Blomberg, Mark Burrell, Greg Guest. 2002. An Ethnographic Approach to Design. The human-computer interaction handbook: fundamentals, evolving technologies and emerging applications. L. Erlbaum Associates Inc., 964-986.

[33] DJ Feil-Seifer, MJ Matarić. 2011. Ethical Principles for Socially Assistive Robotics. IEEE Robot. Autom. Mag., vol. 18, no. 1, 24-31.

[34] Michael Goodrich, Alan Schultz. 2007. Human-Robot Interaction: A Survey. Foundations and Trends in Human-Computer Interaction. 1. 203-275.
[35] Werner Sperschneider, Kirsten Bagger. 2000. Ethnographic fieldwork under industrial constraints: Towards Design-in-Context. International Journal of Humancomputer Interaction - IJHCI.

[36] Virginia Braun, Victoria Clarke. 2006. Using thematic analysis in psychology, Qualitative Research in Psychology, 3:2, 77-101.

[37] Hugh Beyer, Karen Holtzblatt. 1999. Contextual Design. interactions. 6. 32-42.

[38] Jari Friis Jørgensen. 2019. Introduktion til arbejdsgangsanalyse og design. In Sølvkjær, Bergstedt. Sundhedsteknologi i praksis. FADL's Forlag, Copenhagen. 207-230

[39] Bonnie Nardi, Vicki O’Day. 1999. Information Ecologies, Using Technology with Heart. The MIT Press, USA. 